\title{
Coupled Scholte modes supported by soft elastic plates in water
}

\author{
B. M. Staples ๑, T. J. Graham ๑), A. P. Hibbins ๑ , and J. R. Sambles \\ University of Exeter, Exeter EX4 4QL, United Kingdom
}

(Received 30 November 2020; revised 24 March 2021; accepted 2 April 2021; published 11 June 2021)

\begin{abstract}
Localized acoustic surface waves supported by a "soft" elastic plate in water are explored. Unlike many materials, such as aluminum, for soft interfaces the Scholte wave, a localized interface wave, has a speed well below that of sound in water, and the energy of the Scholte wave is no longer mainly localized to the water. We note that the Scholte velocity is largely independent of Poisson's ratio in the solid, and rather than the bulk speeds of sound, the ratio between the Young's modulus and the density of the solid may better indicate whether an interface is soft. The behavior of the coupled Scholte modes along a thin plate with soft interfaces are investigated. It is demonstrated, and experimentally verified using acrylic plates underwater, that for soft interfaces, the symmetric coupled Scholte mode exhibits dispersive behavior, and deviates from the Scholte and the fluid velocities at low frequencies.
\end{abstract}

DOI: 10.1103/PhysRevE.103.063002

\section{INTRODUCTION}

The Scholte mode is a localized, trapped acoustic surface wave that propagates along the boundary between a fluid and an elastic solid. It evanescently decays away from the interface, and propagates along it with a phase velocity that is less than the bulk speeds of sound in both the fluid and the solid [1-3]. For "hard" solid-fluid interfaces, such as "metal-fluid," the Scholte phase velocity is approximately equal to the speed of sound in the fluid. This is often attributed to the fact that the speed of sound in the fluid is substantially less than both the longitudinal and transverse speeds of sound in the metal $[4,5]$.

In this case, the energy of the Scholte wave is mainly localized within the fluid, and its character is dictated by the fluid properties. This has been misconceived as a general property of the Scholte mode in numerous studies [6,7], rather than being indicative of a hard solid-fluid interface [8]. Hence, its application is often overlooked in favor of other modes, despite being the only acoustic surface wave possible for any solid and fluid parameters [4,9], and the only acoustic surface wave that is truly localized on a solid-fluid interface.

Those who already utilize underwater acoustics, for instance in sonar sensors used by the fishing industry, the military, and oil companies, could find benefits from an increased understanding of how underwater sound waves interact with solid materials. Further benefits are possible for the applications that already specifically utilize acoustic surface waves and acoustic modes in a single layer (leaky Lamb modes) [10]. These span over a broad range of disciplines, including biotechnologies and chemistry, where acoustofluidic chips use acoustic surface waves to manipulate microparticles and fluids [11]. Further, civil engineers, the product manufacturing industry, seismologists, and marine geologists use acoustic surface waves to extract information about a material's properties and defects via nondestructive evaluation $[6,12]$.
The behavior of the Scholte wave can be compared, in some measure, with that of surface plasmons. These are collective oscillations of free electrons forming a bound wave that propagates along the interface between a metal and a dielectric [13,14]. The expansive field of plasmonics is largely dedicated to understanding and utilizing their properties in the optical domain [15]. Drawing a comparison between these two surface waves brings to light the potential of both transferable concepts and uses.

On thin metal films, surface plasmons interact, forming two coupled interface modes: a symmetric and antisymmetric pair [16]. This is also true for Scholte modes on a thin plate. For a hard plate, the symmetric coupled Scholte mode or S mode, whose displacement amplitude is symmetric about the center of the plate, appears to be nondispersive, with a phase velocity approximately equal to that of the surrounding fluid $[17,18]$. As a result, many studies neglect its existence, reporting only a single antisymmetric coupled Scholte mode, referred to as the quasi-Scholte mode or A mode [19-22].

For "soft" solid-fluid interfaces, the phase velocity of the Scholte interface wave is notably less than the speed of sound in the fluid [5], with the symmetric coupled Scholte mode on a soft plate exhibiting dispersive behavior, and deviating from the Scholte and the fluid velocities at low frequencies. This has been previously observed by optically exciting these nearfield modes in plastic films, with thicknesses of $130 \mu \mathrm{m}$ [23] and $50 \mu \mathrm{m}$ [24] using laser ultrasonic techniques at megahertz frequencies.

This present study explores these soft solid-fluid interfaces, and examines the conditions under which they occur. The role of the material's elastic properties are fully considered, as opposed to just the speeds of sound [3-5,8,21,22,25]. The behavior of the coupled Scholte modes along a thin plate with soft interfaces is investigated. A simple purely acoustic method was used to directly excite the coupled Scholte modes along acrylic plates of thicknesses 5, 10, and $20 \mathrm{~mm}$ 
underwater, experimentally demonstrating the dispersive behavior of the symmetric Scholte mode, characterized previously for soft films [23,24].

\section{ACOUSTIC MODES OF A PLATE}

In a fluid, bulk sound propagates with speed $c_{\mathrm{F}}$ in the form of longitudinal waves. For isotropic solids, bulk sound propagation can be decomposed into two waves, one longitudinal, and one transverse, that travel with different speeds $c_{\mathrm{L}}$ and $c_{\mathrm{T}}$, respectively [26]. These can be represented by plane waves in the following form:

$$
\begin{aligned}
\phi_{\mathrm{F}}(x, z, t) & =A_{\mathrm{F}} e^{i\left(k_{\mathrm{x}} x+k_{\mathrm{F}, \mathrm{z}} z-\omega t\right)}, \\
\phi_{\mathrm{S}}(x, z, t) & =A_{\mathrm{L}} e^{i\left(k_{\mathrm{x}} x+k_{\mathrm{L}, \mathrm{z}} z-\omega t\right)}, \\
\psi_{\mathrm{S}}(x, z, t) & =\boldsymbol{A}_{\mathrm{T}} e^{i\left(k_{\mathrm{x}} x+k_{\mathrm{T}, \mathrm{z}} z-\omega t\right)} .
\end{aligned}
$$

The scalar potentials $\phi_{\mathrm{F}}$ and $\phi_{\mathrm{S}}$ correspond to the longitudinal waves in the fluid and the solid, respectively, and $\psi_{\mathrm{S}}$ is the vector potential corresponding to the transverse wave in the solid.

Localized surface waves at a solid-fluid interface result from the interaction between these three waves, which have the same angular frequency $\omega=c_{\mathrm{j}}\left|\mathbf{k}_{\mathbf{j}}\right|(j=L, T, F)$ and wave-vector component $k_{\mathrm{x}}$, where Cartesian coordinate $x$ is parallel to the interface, as in Fig. 6. These parameters are purely real for bound Scholte modes when viscoelastic damping is neglected, and are related via

$$
k_{\mathrm{x}}^{2}=\frac{\omega^{2}}{c_{\mathrm{j}}^{2}}-k_{\mathrm{j}, \mathrm{z}}^{2}=\frac{\omega^{2}}{c_{\mathrm{j}}^{2}}+K_{\mathrm{j}, \mathrm{z}}^{2} .
$$

Here $K_{\mathrm{j}, \mathrm{z}}=i k_{\mathrm{j}, \mathrm{z}}$.

These three waves must also satisfy the boundary conditions that the normal component $u_{\mathrm{z}}$ of the displacement vector $\boldsymbol{u}$,

$$
\boldsymbol{u}=\nabla \phi+\nabla \times \boldsymbol{\psi}
$$

the normal stress $\sigma_{\mathrm{zz}}$,

$$
\sigma_{\mathrm{zz}}=\lambda\left(\frac{\partial u_{\mathrm{x}}}{\partial x}+\frac{\partial u_{\mathrm{z}}}{\partial z}\right)+2 \mu \frac{\partial u_{\mathrm{z}}}{\partial z},
$$

and the shear stress $\sigma_{\mathrm{xz}}$,

$$
\sigma_{\mathrm{xz}}=2 \mu\left(\frac{\partial u_{\mathrm{x}}}{\partial z}+\frac{\partial u_{\mathrm{z}}}{\partial x}\right),
$$

must be continuous across the solid-fluid interface. Here $\nabla$ is the vector differential operator, and Lamé's parameters $\lambda$ and $\mu$ describe the elastic properties of a given material.

The modes of a plate of thickness $d$ are the frequency $f=$ $2 \pi \omega$, and wave vector $k_{\mathrm{x}}$, combinations that satisfy the boundary conditions at both of the plate's interfaces, forming a set of six linear equations that dictate the relative wave amplitudes. The determinant of the coefficients of the amplitudes is used to produce two characteristic equations for the symmetric and antisymmetric solutions, as derived by Osborne and Hart [17]. These are given below for the symmetric case:

$$
\begin{aligned}
& {\left[\left(K_{\mathrm{T}, \mathrm{z}}^{2}+k_{\mathrm{x}}^{2}\right)^{2} \operatorname{coth}\left(K_{\mathrm{L}, \mathrm{z}} d / 2\right)-4 k_{\mathrm{x}}^{2} K_{\mathrm{L}, \mathrm{z}} K_{\mathrm{T}, \mathrm{z}} \operatorname{coth}\left(K_{\mathrm{T}, \mathrm{z}} d / 2\right)\right]} \\
& \quad+\frac{\rho_{\mathrm{F}} K_{\mathrm{L}, \mathrm{z}} \omega^{4}}{\rho_{\mathrm{S}} K_{\mathrm{F}, \mathrm{z}} c_{\mathrm{T}}^{4}}=0
\end{aligned}
$$

while for the antisymmetric case:

$$
\begin{aligned}
& {\left[\left(K_{\mathrm{T}, \mathrm{z}}^{2}+k_{\mathrm{x}}^{2}\right)^{2} \tanh \left(K_{\mathrm{L}, \mathrm{z}} d / 2\right)-4 k_{\mathrm{x}}^{2} K_{\mathrm{L}, \mathrm{z}} K_{\mathrm{T}, \mathrm{z}} \tanh \left(K_{\mathrm{T}, \mathrm{z}} d / 2\right)\right]} \\
& +\frac{\rho_{\mathrm{F}} K_{\mathrm{L}, \mathrm{z}} \omega^{4}}{\rho_{\mathrm{S}} K_{\mathrm{F}, \mathrm{z}} c_{\mathrm{T}}^{4}}=0 .
\end{aligned}
$$

Here $\rho_{\mathrm{F}}$ and $\rho_{\mathrm{S}}$ are the densities of the fluid and solid, respectively. The first two terms are equivalent to Lamb's original equations for modes of a free plate [10]. The final term gives the influence of fluid loading, and allows for the additional solutions corresponding to coupled Scholte modes. For hard plates, the fluid-loading term generally acts as a small perturbation. However, for soft plates, this term often has a significant effect on the topology of the spectrum compared to that of a free plate [27].

The modes of the plate can either be obtained using root finding techniques outlined by Lowe [28-30] to find the solutions of these equations, or using the spectral collocation method [31,32], in which the plate is discretized and the governing differential equations are solved via numerical interpolation. The present study exclusively considers the two lowest order modes, corresponding to the symmetric and antisymmetric coupled Scholte modes, with the two techniques yielding indistinguishable results.

\section{THE SCHOLTE MODE}

In this study, a hard interface is defined as being one in which the Scholte velocity is approximately equal to the speed of sound in the fluid, and a soft interface one where the Scholte velocity is notably less than the speed of sound in the fluid. The Scholte velocity is a root of the Scholte-Stoneley equation $[2,4,33,34]$, which can be found by satisfying the boundary conditions across a single solid-fluid interface

$$
\begin{gathered}
\sqrt{1-\frac{c^{2}}{c_{\mathrm{F}}^{2}}}\left[-4 \sqrt{1-\frac{c^{2}}{c_{\mathrm{L}}^{2}}} \sqrt{1-\frac{c^{2}}{c_{\mathrm{T}}^{2}}}+\left(2-\frac{c^{2}}{c_{\mathrm{T}}^{2}}\right)^{2}\right] \\
+\frac{\rho_{\mathrm{F}}}{\rho}\left(\frac{c^{2}}{c_{\mathrm{T}}^{2}}\right)^{2} \sqrt{1-\frac{c^{2}}{c_{\mathrm{L}}^{2}}}=0
\end{gathered}
$$

Here $c=\omega / k_{\mathrm{x}}$ is the phase velocity of the interface wave of a given root, which when purely real and $0<c<$ $\min \left(c_{\mathrm{F}}, c_{\mathrm{T}}, c_{\mathrm{L}}\right)$ corresponds to the Scholte velocity $c_{\mathrm{sch}}$.

Many studies have investigated how the Scholte velocity changes with the ratio of the densities, with the bulk speeds of sound, and with the Rayleigh velocity, which itself depends on the bulk speeds of sound in the solid $[8,21,22,25]$. Others adopted a simplified view that a soft interface is one where the transverse speed of sound in the solid is less than the speed of sound in the fluid $\left(c_{\mathrm{T}}<c_{\mathrm{F}}\right)$, with interfaces not meeting this criteria being hard [3-5]. This approximation is valid for many homogeneous isotropic solid-fluid interfaces, but it is not without exception (see Fig. 1). However, from Eq. (8), it follows that the Scholte velocity depends on two parameters of the fluid, and three parameters of the solid. Further, many 


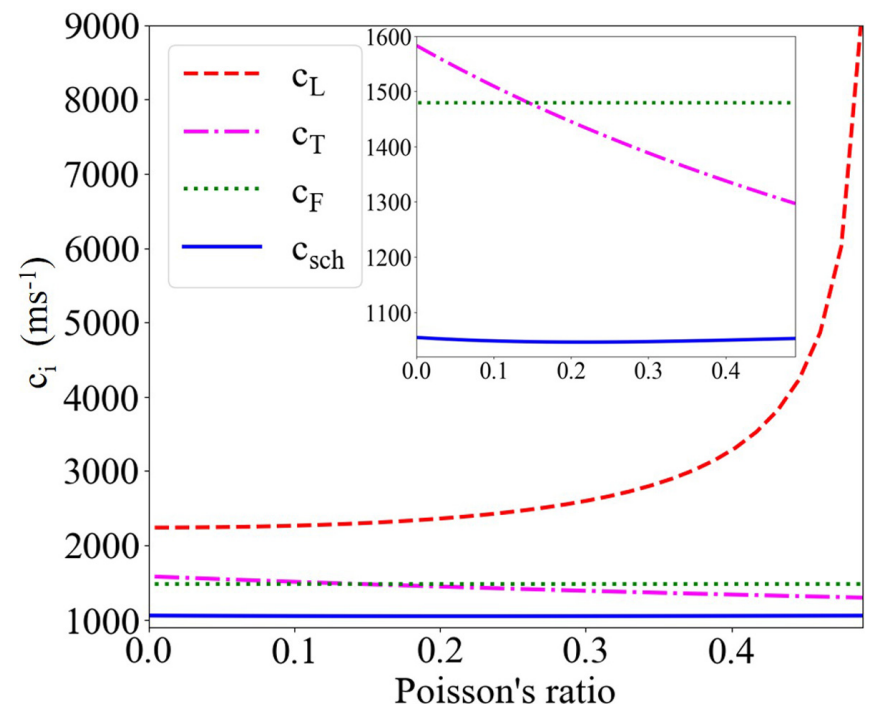

FIG. 1. Variation in the Scholte velocity $c_{\text {sch }}$, and the transverse and longitudinal bulk speeds of sound $c_{\mathrm{T}}$ and $c_{\mathrm{L}}$ over the allowed range of positive Poisson's ratio $v$, while the Young's modulus $E$, solid density $\rho$, and fluid parameters remain constant. The region over which $c_{\mathrm{T}}$ and $c_{\text {sch }}$ vary is expanded in the inset.

investigations fail to consider that the bulk speeds of sound are not independent of the densities, which are given by

$$
\begin{gathered}
c_{\mathrm{L}}=\sqrt{\frac{\lambda+2 \mu}{\rho}}=\sqrt{\frac{E(1-v)}{\rho(1+v)(1-2 v)},} \\
c_{\mathrm{T}}=\sqrt{\frac{\mu}{\rho}}=\sqrt{\frac{E}{2 \rho(1+v)}}, \\
c_{\mathrm{F}}=\sqrt{\frac{\lambda_{\mathrm{F}}}{\rho_{\mathrm{F}}}}=\sqrt{\frac{K}{\rho_{\mathrm{F}}}} .
\end{gathered}
$$

The Lamé's parameters can be replaced, with the elastic properties of the solid described by the Young's modulus $E$ and Poisson's ratio $v$, while the fluid is described entirely by the bulk modulus $K$. In investigating the effects of these material properties on the Scholte velocity, we found that, contrary to common convention, the use of the speeds of sound may not be the best indicator of the value of the Scholte velocity.

Figure 1 shows how the Scholte velocity and bulk speeds of sound in the solid vary with Poisson's ratio for a fixed Young's modulus, solid density, and fluid parameters. The longitudinal speed of sound varies significantly with Poisson's ratio, and the inserted expansion of the graph highlights the noticeable variation of the transverse speed of sound in the solid, going from greater than to less than the fluid sound velocity over the range of $v$. However, the Scholte velocity exhibits negligible change with Poisson's ratio, varying over a range of less than $8 \mathrm{~ms}^{-1}$, with similar results achieved for different values of the fixed parameters. In making the approximation that the Scholte velocity is independent of Poisson's ratio, its value is now effectively determined by two independent parameters of the solid, the density and the Young's modulus. Therefore, for a given fluid, it is probably better to use these parameters, rather than the bulk speeds of sound, to indicate the value of the Scholte velocity relative to the fluid sound velocity.

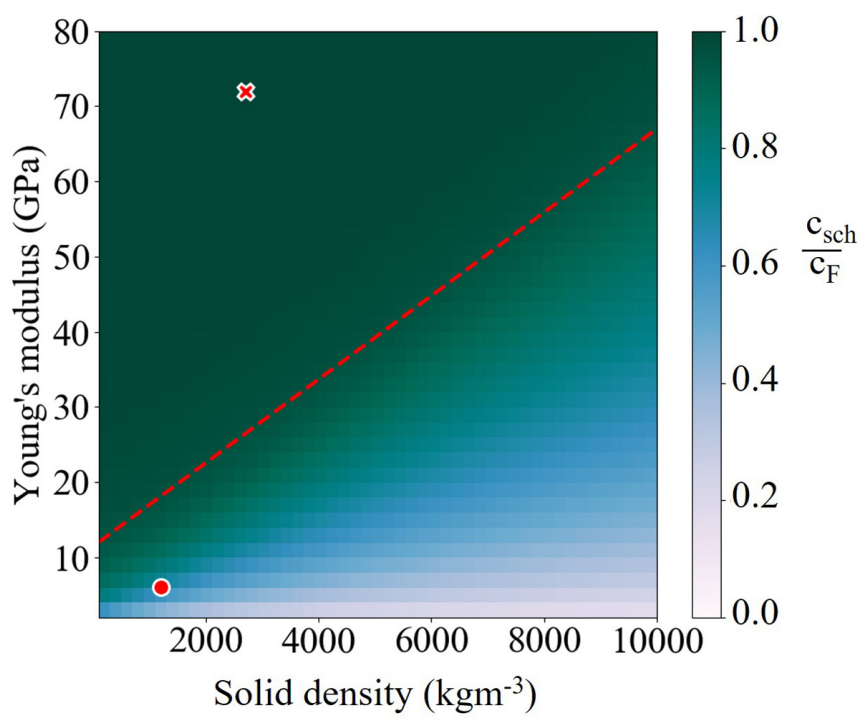

FIG. 2. Color plot showing the variation of the Scholte velocity $c_{\text {sch }}$ with respect to a fixed fluid sound velocity $c_{\mathrm{F}}$ over a range of the Young's modulus $E$ and the density $\rho$ of the solid. The arbitrary fixed Poisson's ratio is $v=0.33$, and fluid properties are that of water $\left(c_{\mathrm{F}}=1480 \mathrm{~ms}^{-1}, \rho_{\mathrm{F}}=1000 \mathrm{~kg} \mathrm{~m}^{-3}\right)$. The red dashed line approximately indicated $c_{\mathrm{sch}}=0.95 c_{\mathrm{F}}$. The Young's modulus and density of aluminum is given by the red cross (above the red dashed line, hard example), and of acrylic by the red dot (below the red dashed line, soft example).

Given that Poisson's ratio describes the expansion of a material perpendicular to the axis along which it is compressed, we can physically interpret its primary effect on the Scholte wave as being the amount the solid displaces in the $x$ direction in response to a displacement in the $z$ direction caused by the fluid. On analyzing the displacement fields of the Scholte wave with varying Poisson's ratio, it is confirmed that the eccentricity of the elliptical particle trajectories is significantly affected, while the wavelength at the interface is not.

Figure 2 illustrates how the ratio between the Young's modulus and the density of a solid indicates the proximity of the Scholte velocity to the fluid sound velocity for a watersolid interface, with the red dashed line at approximately $c_{\mathrm{sch}}=0.95 c_{\mathrm{F}}$ arbitrarily separating the regions of hard and soft interfaces. Solids with larger densities lead to slower interface waves, while increasing the Young's modulus brings the Scholte speed closer to the speed of sound in the fluid. In other words, as the readiness of the solid to deform decreases, the surface wave becomes increasingly localized to the fluid. It follows that for "softer" interfaces, with slower Scholte velocities, the mode penetrates deeper into the solid, contradicting the widely quoted belief that the energy of the Scholte mode is predominantly localized to the fluid $[6,7,35]$.

A large number of studies considering underwater acoustics and solid materials explored either metal or plastic, as these materials are widely used in applications, and many can be considered homogeneous and isotropic. The assumption that metal-water interfaces are hard, while plastic-water interfaces are soft is supported by Fig. 2, where the red cross in the hard region corresponds to the Young's modulus and density of aluminum, and the red dot in the soft region corresponds to 

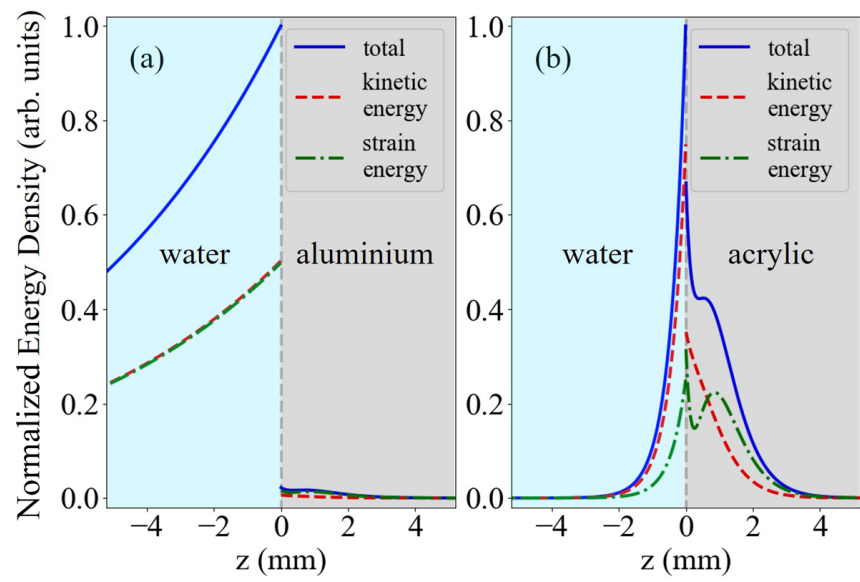

FIG. 3. Total energy density of the Scholte wave across the interface between water and (a) aluminum (hard interface), (b) acrylic (soft interface), calculated by summing the kinetic and strain energy densities [36,37].

acrylic. It is evident from this that the high Young's moduli, arising from strong metallic bonding, dictates that metal-water interfaces are likely to be hard. Despite the obviously lower density of plastics, it is the comparatively much weaker intermolecular forces, and hence significantly lower Young's moduli, that means plastic-water interfaces tend to be soft.

Figure 3(a) shows the energy distribution of the Scholte mode across an aluminum-water interface. Here the Scholte velocity is approximately equal to the speed of sound in water, and consequently the decay length in the water is long, the energy is localized to the fluid, and the wave behaves approximately like a grazing wave in the water, with the kinetic and potential (strain) energy density contributions in the fluid being approximately equal. By contrast, the energy distribution across an acrylic-water interface in Fig. 3(b) contradicts the notion that the Scholte wave is predominantly fluid-borne, and confirms Guzhev's theory [8] that a redistribution of the energy density from the fluid to the solid medium occurs as the solid-fluid interface becomes increasingly soft. The shape of the energy distribution in the solid occurs due to the components of displacement (used in the calculations of the velocity, stress, and strain) being composed of two opposing contributions from the transverse and longitudinal partial waves, each with different decay lengths.

\section{THE COUPLED SCHOLTE MODES}

We now go on to explore coupled Scholte modes in thin plates. The dispersion curve for the antisymmetric coupled Scholte waves propagating underwater along an aluminum plate is shown in Fig. 4(a). The symmetric mode has been omitted, but would follow the line corresponding to the speed of sound in water, $c_{\mathrm{F}}$. The dispersion for an acrylic plate underwater is shown as an example of a soft plate in Fig. 4(b), with the Scholte phase velocity notably below the water sound line. This allows the higher energy solution corresponding to the symmetric coupled Scholte mode to be clearly visible, as opposed to being indistinguishable from the fluid sound line for aluminum.
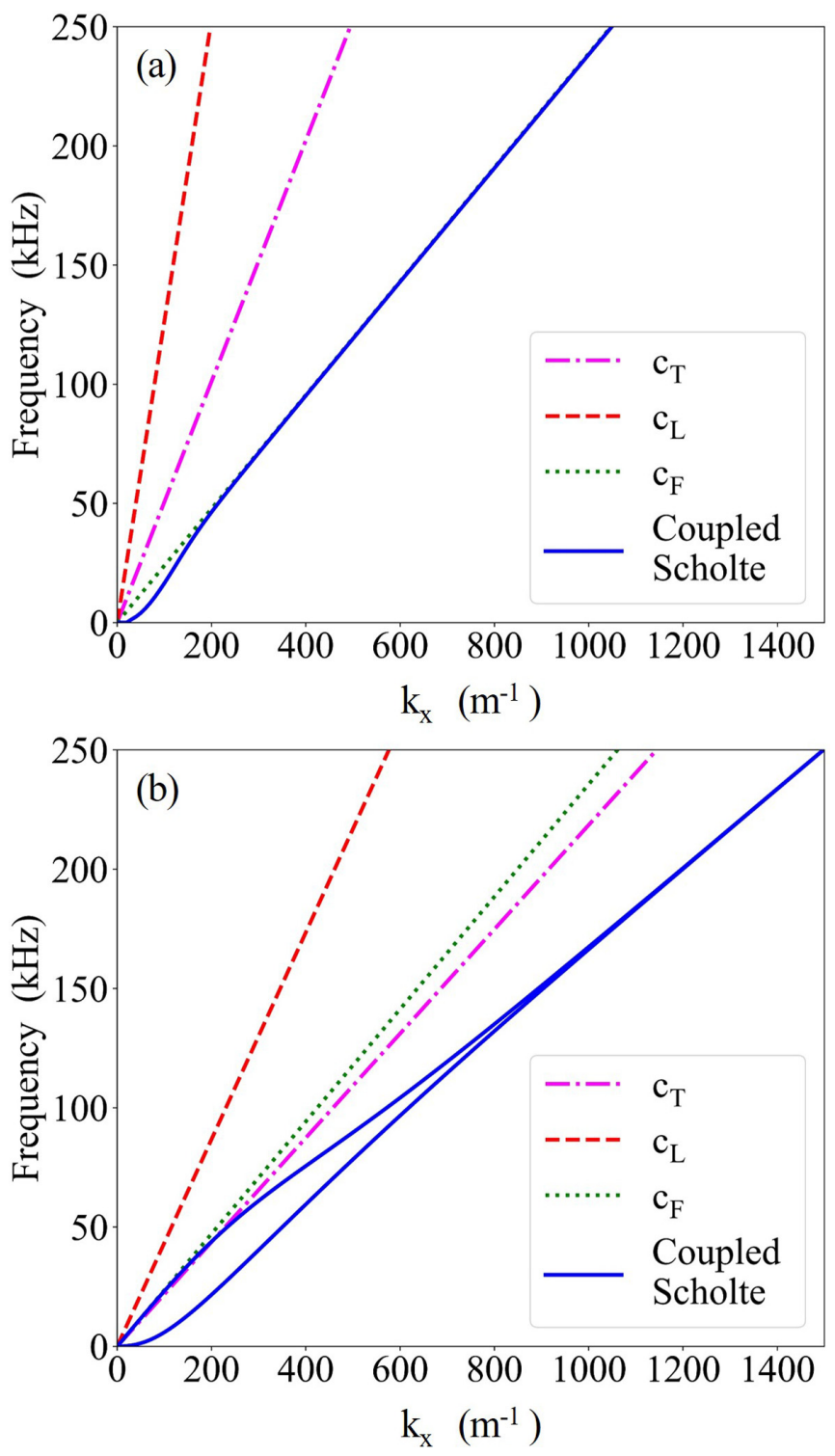

FIG. 4. The dispersion curves for coupled Scholte modes in 10mm-thick plates underwater are shown, together with the bulk speeds of sound in the system. (a) For an aluminum plate the antisymmetric coupled Scholte mode is shown, and the symmetric mode that follows $c_{\mathrm{F}}$ is omitted. This is an example of a hard plate. (b) For an acrylic (soft) plate, an additional symmetric coupled Scholte mode is clearly present, as well as the lower frequency antisymmetric mode.

For both plates, the dispersion curves have a higher inplane wave vector than that of a grazing sound wave in the water (the water sound line), indicating from Eq. (2) that $K_{\mathrm{F}, \mathrm{z}}$ is real, and the wave-vector component perpendicular to the interface is purely imaginary. Consequently the wave's amplitude decays away from the plate, and there is no propagation of the wave through the water in the $z$ direction. Except for the lowest wave vectors, the mode on aluminum propagates with a phase velocity very close to $c_{\mathrm{F}}$, the magnitude of $K_{\mathrm{F}, \mathrm{z}}$ is very small, and the decay length in the liquid is correspondingly large. For acrylic, however, the coupled Scholte mode is slower, and more tightly bound to the plate. 
For high frequencies, the two characteristic equations approach the same limit, and hence the two curves converge. This is readily understood, for on increasing the frequency, the decay length within the solid decreases, and the two surface modes eventually become independent, effectively identical, bulk solid interface modes. And the energy becomes increasingly localized to the interface, as shown for acrylic in Fig. 5(a).

For the acrylic plate at lower wave vectors, there is a strong coupling of the Scholte modes on each interface, resulting in two distinct dispersive coupled mode solutions. The lower energy solution has particle displacement that is antisymmetric about the plate center, as demonstrated by the displacement grid inset in Fig. 5(b). The higher energy mode is the symmetric coupled Scholte mode, and unlike in the hard case, displays significant dispersive behavior, in agreement with previous studies on soft films [23,24]. The dispersion agrees with Osborne and Hart's [17] analysis of the characteristic functions, predicting that at low frequency (wavelength is long by comparison with the plate thickness) the phase and group velocities of the symmetric mode approach those of the sound in the fluid, while the phase and group velocities of the antisymmetric mode approach zero.

Visualizing these strongly coupled modes using the exaggerated displacement grids in Figs. 5(b) and 5(c), it is intuitive that the bending antisymmetric mode be a lower energy solution than that of the periodic expansion and compression of the symmetric mode. For the antisymmetric mode in Fig. 5(b), the particles along the center of the plate display a shearing motion that approximates to slow free wave behavior. This is evident from the closeness in values of the kinetic and strain energy contributions in the central region of the plate. The total energy density is largely localized to the plate, and as this mode sits further from the water sound line, it decays more quickly away from the plate.

For the symmetric mode in Fig. 5(c), the $z$ component of displacement is zero at the plate's center. As frequency decreases, the thickness of the plate becomes very small relative to the wavelength, and the normal displacement at the interface decreases. Given that the component of displacement parallel to the interface becomes increasingly dominant, and the fluid only supports longitudinal waves, it follows that the behavior of the wave in the water begins to approximate that of a grazing bulk wave. Hence the phase velocity of the symmetric coupled Scholte mode tends to the speed of sound in water and the energy becomes increasingly localized to the fluid.

\section{EXPERIMENTAL VERIFICATION}

The experimental setup is illustrated in Fig. 6. A Neptune Sonar D70 transducer was used, which is omnidirectional to a good approximation. It was placed almost in contact with the edge of a small hole in the acrylic plate's surface. The diffraction of the pressure field through the hole provides the high momentum components needed to excite the Scholte modes.

Ultrasonic broadband pulses were used to excite the plate, at a central frequency of $125 \mathrm{kHz}$. The transmitted pulse was detected by a Precision Acoustics 1-mm needle hydrophone.
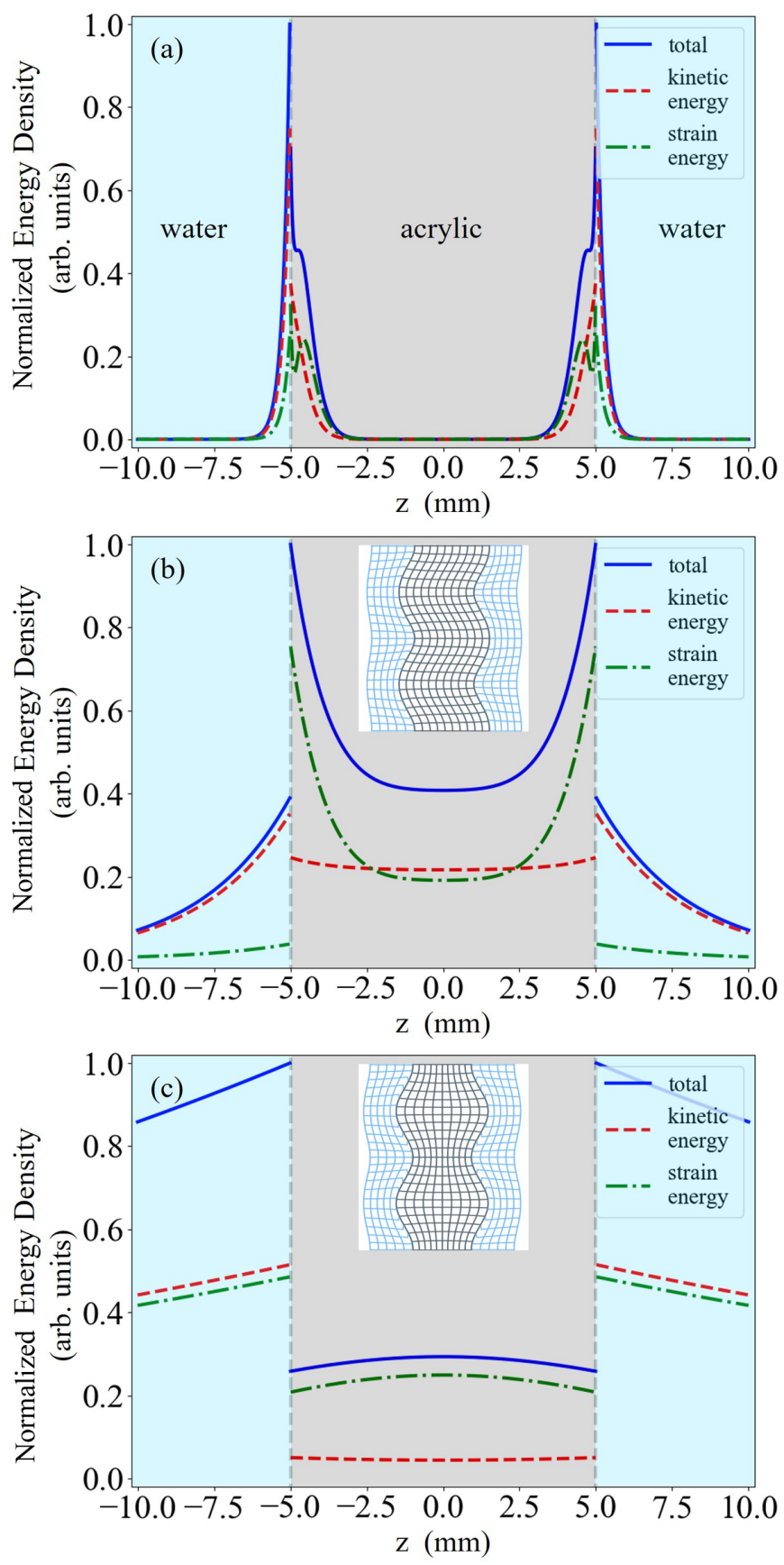

FIG. 5. Total energy density for the coupled Scholte mode across a 10 -mm-thick acrylic plate in water, calculated by summing the kinetic and strain energy densities [36,37]. (a) shows the distribution for a weakly coupled, high frequency Scholte mode, while (b) and (c) show the distribution for strongly coupled Scholte modes at $f=$ $20 \mathrm{kHz}$ for the slower antisymmetric and faster symmetric mode, respectively (indicated by the inset displacement grids).

This was scanned spatially along the plate, at a distance of $0.5 \mathrm{~mm}$ away from the surface, allowing the near-field signal to be detected. At each point of the spatial scan the variation of signal in time was recorded, which is equivalent to recording the acoustic pressure amplitude. The amplitude and phase for each frequency at each point were obtained by Fourier transforming the time-dependent data. Then the 


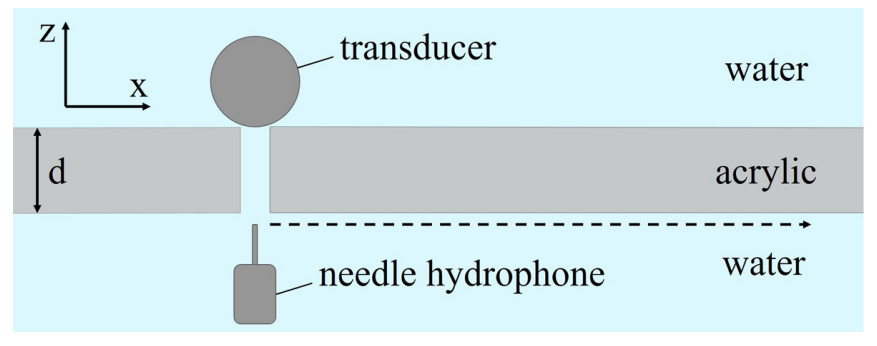

FIG. 6. A schematic of the experimental setup used to excite and detect coupled Scholte modes in acrylic plates of thicknesses $d=5$, 10 , and $20 \mathrm{~mm}$ underwater.

wave-vector spectrum for every frequency was obtained by a spatial Fourier transform.

The experimental results are presented in Fig. 7, with the theoretical dispersion curves also shown. As we are only interested in the coupled Scholte modes, the data for the low wave vectors on the radiative side of $c_{\mathrm{F}}$ have been removed, although some signal along the sound line remains. Variations in the amplitude of the modes can be observed due to variations in the source output, detector sensitivity, and the unknown coupling efficiency between the source and excited modes. These fluctuations have been minimized by normalizing the data to the integral of amplitudes at each frequency, with the maximum normalized amplitude at each frequency being set to 1 . To further emphasize the mode position, signals less than the mean background noise for high momentum values have been set to zero. The symmetric coupled Scholte mode is clearly demonstrated, most prominently by the thinnest plate, Fig. 7(a). Increasing the thickness of the plate in Fig. 7(b) weakens the coupling between the interfaces, and the two modes converge to the dispersion of the Scholte mode for a single interface. The clear nondispersive behavior of the modes after converging at high $k_{\mathrm{x}}$ is illustrated by the thickest plate in Fig. 7(c).

The theoretical curves were fit to the data, and from this the Young's modulus of the acrylic was extracted, and determined to be $5.93 \pm 0.09 \mathrm{GPa}$. When considering the Young's modulus of polymers, it is important to note that due to relaxation phenomena, the Young's modulus for viscoelastic materials $[38,39]$, such as acrylic, significantly reduce at low frequencies. Consequently, values at ultrasonic frequencies can exceed those obtained using zero frequency techniques by as much as a factor of 10 [40]. This frequency dependence of the elastic properties of acrylic is most significant at low frequencies [41]. The values vary negligibly over ultrasonic frequencies $[42,43]$, and by extension the frequency range, $10-250 \mathrm{kHz}$, used in this experiment. Our value sits within the range of Young's moduli extracted from the speeds of sound given for acrylic 4.59-6.32 GPa [24,44,45]. As expected, this significantly differs from the zero frequency literature value of the Young's modulus 3.30 GPa [46], and the manufacturer's data sheet value of $3.21 \mathrm{GPa}$.

The theory of viscoelasticity also describes the dissipation of acoustic energy in the form of heat, by including damping terms in the equations that describe the propagation of sound through the solid. In our theoretical analysis, the damping term was neglected. Although it is clear from the extracted
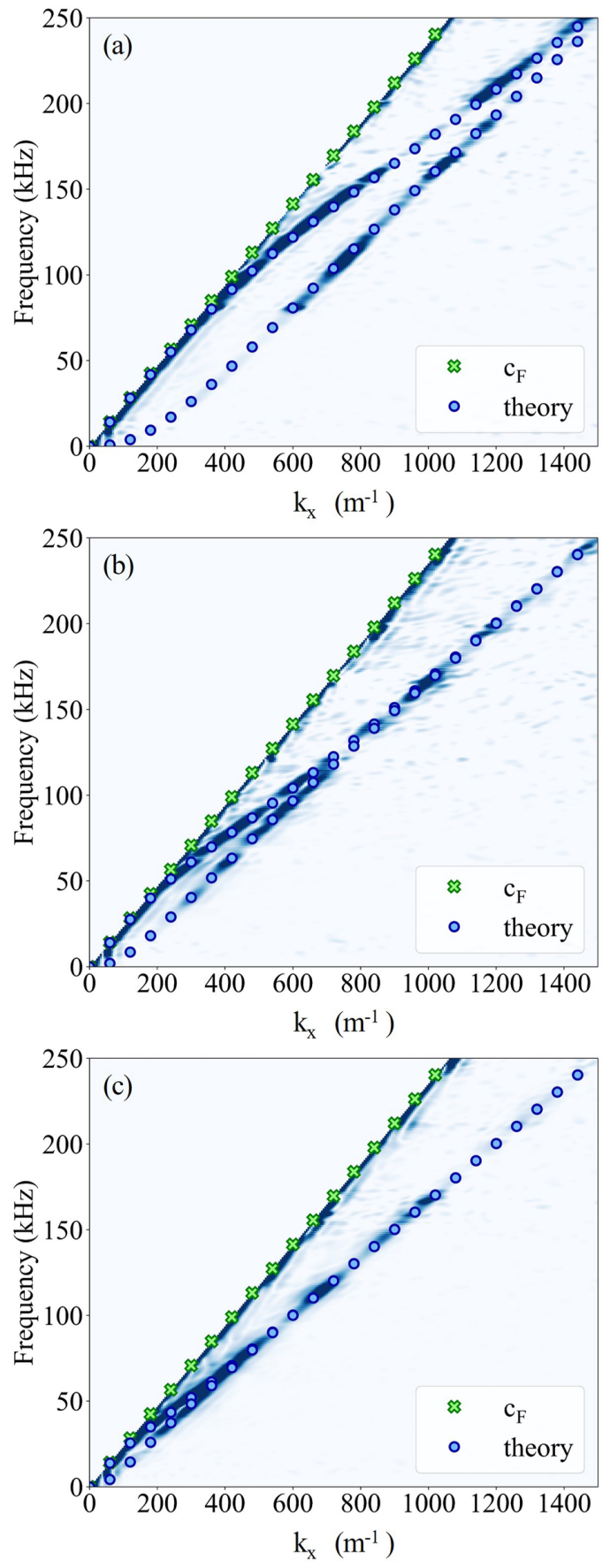

FIG. 7. Experimental data obtained for acrylic plates of thickness $d=$ (a) $5 \mathrm{~mm}$, (b) $10 \mathrm{~mm}$, and (c) $20 \mathrm{~mm}$ are presented as color maps of the Fourier transformed data. The peaks in Fourier amplitude, shown in blue, indicate the dispersion of the modes, in agreement with the overlaid theoretical predictions.

Young's modulus that the effects of viscoelasticity significantly affect the value of the bulk speeds of sound in the solid, as well as the coupled Scholte mode, these effects stand without the explicit inclusion of viscoelastic damping terms in the mathematics. Favretto-Anres [47] found the omission of the damping term altered the value of the Scholte velocity on a single interface by a fraction of a percentage, which is a negligible effect for this purpose. From the high level agreement 
between our experimental and theoretical dispersion curves, we conclude that this is also true for the coupled system, and neglecting damping is a suitable approximation.

\section{CONCLUSIONS}

For a given fluid, the ratio between the Young's modulus and the density of the solid may better indicate the proximity of the Scholte velocity to the fluid sound velocity, compared to using the bulk speeds of sound and the density. This is in part due to the dependence of the bulk speeds of sound on the density of the solid. More significantly, it is shown that the Scholte velocity is approximately independent of Poisson's ratio, reducing the number of solid parameters that the Scholte velocity depends upon.

It is highlighted that for soft interfaces, where the Scholte velocity is notably less than the speed of sound in water, the energy density of the Scholte mode is localized more to the solid. This is at variance with the lingering misconception that the energy of the Scholte mode is localized to the fluid.

For the case of soft plates underwater, both symmetric and antisymmetric coupled Scholte modes exhibit dispersive behavior, whose reduced phase velocity allows both dispersion curves to be clearly distinguishable from the fluid sound line. These modes were excited in acrylic plates underwater using ultrasonic pulses, and the dispersion obtained from the experimental data verifies their existence, and demonstrates an excellent fit to the theory.

In fitting the theoretical curve to the experimental data, we conclude that the inclusion of a damping term to describe the dissipation of acoustic energy as heat has a negligible effect on the shape of the dispersion curves for both coupled Sholte mode symmetries. Although the viscoelastic damping terms can be justifiably omitted, the effects of viscoelasticity cannot be ignored for materials such as polymers, as the elastic parameters, and hence the velocities of sound in the media may have a strong frequency dependence. However, there is no evidence that the parameters for acrylic vary over the range of frequencies used in this experiment.

All data created during this research are openly available from the University of Exeter's institutional repository [48].

\section{ACKNOWLEDGMENTS}

The authors would like to acknowledge financial support from the Engineering and Physical Sciences Research Council (EPSRC) of the United Kingdom, via the EPSRC Centre for Doctoral Training in Metamaterials and Thales UK Limited.
[1] J. G. Scholte, The range and existence of Rayleigh and Stoneley waves, Mon. Not. R. Astron. Soc. Geophys. Suppl. 5, 120 (1947).

[2] J. H. Ansell, The roots of the Stoneley wave equation for solidliquid interfaces, Pure Appl. Geophys. 94, 172 (1972).

[3] F. Padilla, M. de Billy, and G. Quentin, Theoretical and experimental studies of surface waves on solid-fluid interfaces when the value of the fluid sound velocity is located between the shear and the longitudinal ones in the solid, J. Acoust. Soc. Am. 106, 666 (1999).

[4] C. Glorieux and K. Van de Rostyne, On the character of acoustic waves at the interface between hard and soft solids and liquids, J. Acoust. Soc. Am. 110, 1299 (2001).

[5] S. Nasr, J. Duclos, and M. Leduc, Scholte wave characterization and its decay for various materials, J. Acoust. Soc. Am. 87, 507 (1990).

[6] F. B. Cegla, P. Cawley, and M. J. S. Lowe, Material property measurement using the quasi-Scholte mode-A waveguide sensor, J. Acoust. Soc. Am. 117, 1098 (2005).

[7] H. Uberall, Repulsion of phase-velocity dispersion curves and the nature of plate vibrations, J. Acoust. Soc. Am. 96, 908 (1994).

[8] S. N. Guzhev, Study of phase velocity and energy distribution of Stoneley waves at a solid-liquid interface, J. Acoust. Soc. Am. 95, 661 (1994).

[9] P. C. Vinh, Scholte-wave velocity formulae, Wave Motion 50, 180 (2013).

[10] H. Lamb, On waves in an elastic plate, Proc. R. Soc. A 93, 114 (1917).

[11] V. Aubert, A simple acoustofluidic chip for microscale manipulation using evanescent Scholte waves, Lab Chip 16, 2532 (2016).
[12] T. J. Graham, Underwater Acoustic Waves on Structured and Unstructured Plates, Ph.D. thesis, University of Exeter, 2018.

[13] D. Sarid and W. A. Challener, Modern Introduction to Surface Plasmons: Theory, Mathematica Modeling, and Applications (Cambridge University Press, Cambridge, UK, 2010).

[14] A. D. Boardman, Electromagnetic Surface Modes (WileyInterscience, New York, 1982).

[15] M. I. Stockman, Roadmap on plasmonics, J. Opt. 20, 043001 (2018).

[16] E. N. Economou, Surface Plasmons in Thin Films, Phys. Rev. 182, 539 (1969).

[17] M. F. M. Osborne and S. D. Hart, Transmission, reflection, and guiding of an exponential pulse by a steel plate in water. I. Theory, J. Acoust. Soc. Am. 17, 1 (1945).

[18] J. Sessarego, Two Scholte-Stoneley waves on doubly fluidloaded plates and shells, J. Acoust. Soc. Am. 101, 135 (1997).

[19] F. B. Cegla, Ultrasonic Waveguide Sensors for Fluid Characterisation and Remote Sensing, Ph.D. thesis, Imperial College London, 2006.

[20] A. E. Takiy, Theoretical analysis and experimental validation of the Scholte wave propagation in immersed plates for the characterization of viscous fluids, in 2013 IEEE International Ultrasonics Symposium (IUS) (IEEE, 2013), pp. 1614-1617.

[21] Y. Can Uz, Ultrasound : ICA 2016-741 An ultrasonic waveguide sensor for monitoring alcohol concentration in wateralcohol mixtures, in 22nd International Congress on Acoustics (ICA) (2016).

[22] O. Önen, Dispersion and sensitivity analysis of quasi-Scholte wave liquid sensing by analytical methods, J. Sensors 2017, 1 (2017).

[23] X. Xu, J. Goossens, G. Shkerdin, and C. Glorieux, Effect of loading a plate with different liquids on the propagation 
of Lamb-like waves, IEEE Trans. Ultrason. Ferroelectr. Freq. Control 55, 675 (2008).

[24] K. V. Rostyne, C. Glorieux, W. Gao, W. Lauriks, and J. Thoen, Experimental investigation of leaky Lamb modes by an optically induced grating, IEEE Trans. Ultrason. Ferroelectr. Freq. Control 49, 1245 (2002).

[25] G. D. Meegan, M. F. Hamilton, Y. A. Il'inskii, and E. A. Zabolotskaya, Nonlinear Stoneley and Scholte waves, J. Acoust. Soc. Am. 106, 1712 (1999).

[26] L. M. Brekhovskih, Waves in Layered Media (Academic, New York, 1960).

[27] S. I. Rokhlin, On the topology of the complex wave spectrum in a fluid-coupled elastic layer, J. Acoust. Soc. Am. 85, 1074 (1989).

[28] M. J. S. Lowe, Plate waves for the NDT of diffusion bonded titanium, Ph.D. thesis, Imperial College London, 1992.

[29] M. J. S. Lowe, Matrix techniques for modelling ultrasonic waves in multilayered media, IEEE Trans. Ultrason. Ferroelectr. Freq. Control 42, 525 (1995).

[30] M. J. S. Lowe, Disperse User Manual, 2nd Disperse, Non-destructive testing laboratory (Imperial College London, 2013).

[31] D. A. Kiefer, M. Ponschab, S. J. Rupitsch, and M. Mayle, Calculating the full leaky Lamb wave spectrum with exact fluid interaction, J. Acoust. Soc. Am. 145, 3341 (2019).

[32] A. T. I. Adamou and R. V. Craster, Spectral methods for modelling guided waves in elastic media, J. Acoust. Soc. Am. 116, 1524 (2004).

[33] W. L. Pilant, Complex roots of the Stoneley-wave equation, Bull. Seismol. Soc. Am. 62, 285 (1972).

[34] E. Strick and A. S. Ginzbarg, Stoneley-wave velocities for a fluid-solid interface, Bull. Seismol. Soc. Am. 46, 281 (1956).
[35] M. de Billy and G. Quentin, Experimental study of the Scholte wave propagation on a plane surface partially immersed in a liquid, J. Appl. Phys. 54, 4314 (1983).

[36] K. F. Graff, Wave Motion in Elastic Solids (Clarendon, Oxford, 1975).

[37] B. A. Auld, Acoustic Fields and Waves in Solids (Krieger Publishing Company, Malabar, FL, 1990).

[38] E. A. Campo, Selection of Polymeric Materials (William Andrew Publishing, Norwich, NY, 2008).

[39] J. M. G. Cowie, Polymers: Chemistry and Physics of Modern Materials (Intertext, New York, 1973).

[40] M. Sinhaand D. J. Buckley, Physical Properties of Polymers Handbook (Springer, New York, 2007).

[41] Y. Liao and V. Wells, Estimation of complex modulus using wave coefficients, J. Sound Vibr. 295, 165 (2006).

[42] W. Xia, D. Piras, J. C. G. van Hespen, W. Steenbergen, and S. Manohar, A new acoustic lens material for large area detectors in photoacoustic breast tomography, Photoacoustics 1, 9 (2013).

[43] J. E. Carlson, J. van Deventer, A. Scolan, and C. Carlander, Frequency and temperature dependence of acoustic properties of polymers used in pulse-echo systems, in IEEE Symposium on Ultrasonics (IEEE, 2003), Vol. 1, pp. 885-888.

[44] A. S. Birks, R. E. Green, and P. McIntire, Nondestructive Testing Handbook, 3rd ed., Vol 7: Ultrasonic Testing (American Society for Nondestructive Testing, 1991).

[45] E. Ginzel and B. Turnbull, Determining approximate acoustic properties of materials, e-J. Nondestruct. Test., 12 (2016).

[46] J. Brandrup, Polymer Handbook (Wiley-Interscience, New York, 1989).

[47] N. Favretto-Anres, Theoretical study of the Stoneley-Scholte wave at the interface between an ideal fluid and a viscoelastic solid, Acustica 82, 829 (1996).

[48] https://ore.exeter.ac.uk/. 